\title{
Density-functional versus wave-function methods: Toward a benchmark for the jellium surface energy
}

\author{
Zidan Yan, John P. Perdew, and Stefan Kurth \\ Department of Physics and Quantum Theory Group, Tulane University, New Orleans, Louisana 70118 \\ Carlos Fiolhais and Luis Almeida \\ Center for Computational Physics, University of Coimbra, P3000 Coimbra, Portugal
}

(Received 4 October 1999)

\begin{abstract}
For the surface energy of jellium at alkali-metal densities, the local-density approximation (LDA) and more advanced density-functional methods disagree strongly with the wave-function-based Fermi hypernetted-chain and diffusion Monte Carlo methods. We present a wave-vector interpolation correction to the generalized gradient approximation which gives jellium surface energies consistent with two other estimates based on advanced density functionals. LDA makes compensating errors at intermediate and small wave vectors. Studies of small jellium clusters also support the density-functional estimate for the jellium surface energy.
\end{abstract}

Kohn-Sham spin density-functional theory ${ }^{1}$ is now the most widely used method for electronic structure calculations in solid-state physics and quantum chemistry. Densityfunctional approximations are often constructed nonempirically, starting from the electron gas of uniform or slowly varying density. The simple local-density approximation (LDA) is remarkably accurate for periodic solids, and advanced density functionals, such as generalized gradient approximations $^{2}$ (GGA's) and meta-GGA's, ${ }^{3}$ work well for molecules. Typical errors ${ }^{3}$ in the atomization energies of small molecules are $22 \%$ in LDA, $7 \%$ in GGA, and $3 \%$ in meta-GGA.

Density-functional approximations are being applied to increasingly large and complex systems, but they must be tested against benchmark systems that are small or simple enough to provide an "exact"' ground-state energy, either from experiment or from correlated-wave-function theory. A possible benchmark which is physically different from a bulk solid or a molecule is the jellium surface. The energy required to create the surface of a uniform electron gas (jellium) ought to be described by these density functionals even more accurately than the energy to atomize a molecule. Indeed the three density functionals described above, and several others to be described below, all predict the same jellium surface exchange-correlation energy $\sigma_{x c}$ within a few percent, but show no such agreement with the available wavefunction-based methods: Fermi hypernetted chain ${ }^{4}$ (FHNC) and diffusion Monte Carlo ${ }^{5}$ (DMC) (Table I). The DMC value is about $150 \pm 30 \mathrm{erg} / \mathrm{cm}^{2}$ higher than the LDA value for $2.07 \leqslant r_{s} \leqslant 4$, where $r_{s}$ is the bulk density parameter. The relative difference is $7 \%$ for $r_{s}=2.07$ ("aluminum"), and as much as $50 \%$ for $r_{s}=4$ ("sodium"). If the mutually consistent density functional estimates are correct, then the wavefunction-based estimates need to be reconsidered. If one of the less mutually consistent wave-function-based estimates is correct, then something important is missing from our understanding of density-functional theory.

Popular GGA's provide a negative correction of $3 \%$ or $4 \%$ to the LDA surface exchange-correlation energy. Recently, density-functional approximations more sophisticated than GGA have been developed. The following three ap- proaches yield jellium surface energies $\sigma_{x c}$ (Table I) that agree among themselves to $1 \%$, and are greater than $\sigma_{x c}^{L D A}$ by about $2 \%$ : (1) A meta-GGA, ${ }^{3}$ which makes use not only of the local density and its gradient but also of the orbital kinetic-energy density, and is free of the self-correlation error that bedevils the low-density limits for LDA and GGA. (2) The random-phase approximation, ${ }^{7}$ (RPA) which yields exact exchange and long-range correlation, plus a GGA for the short-range correction to RPA (Refs. 8 and 9) (RPA+). Table I shows the results of an improved construction of this GGA correction, using the real-space cutoff ${ }^{10}$ of the gradient expansion for the correlation hole within and beyond RPA. (3) A new wave-vector interpolation as a long-range correction to GGA exchange and correlation, the subject of this paper, which shows that LDA makes compensating errors at intermediate and small wave vectors. Consistent with these estimates, Skriver and Rosengaard" found that "localdensity theory" ... can provide surface energies which are at least as accurate as those derived from experiments" for real metals. GGA surface energies ${ }^{12}$ are also in good agreement with experiment, even for sodium $\left(r_{s} \approx 4\right)$.

The electron density at a metal surface is radically inhomogeneous, and the surface energy $\sigma$ (work required to create a unit area of new surface) is dominated by exchange and correlation. Yet, since the early work of Lang and Kohn, ${ }^{6}$ surface energies have been calculated within the localdensity approximation (LDA) for exchange and correlation,

$$
E_{x c}^{L D A}[n]=\int d^{3} r n(\mathbf{r}) \varepsilon_{x c}^{u n i f}(n(\mathbf{r})),
$$

which is based upon the uniform electron gas. To understand this situation, Langreth and Perdew ${ }^{13}$ analyzed the surface exchange-correlation energy $\sigma_{x c}$ into contributions from dynamic density fluctuations of various wave vectors $k$ :

$$
\sigma_{x c}=\int_{0}^{\infty} d\left(\frac{k}{2 k_{F}}\right) \gamma_{x c}(k),
$$

where $k_{F}$ is the bulk Fermi wave vector. They argued that LDA is right at large $k$, i.e., for the short-range part of the 
TABLE I. Estimates of the jellium surface exchange-correlation energy $\sigma_{x c}$, in erg $/ \mathrm{cm}^{2} . r_{s}$ is the bulk density parameter, in bohr. All functionals have been evaluated on self-consistent LDA densities. The "new WVI" column is our new wave-vector-interpolation-corrected GGA. The "mrd" is the mean relative deviation of each method from "RPA+," which we regard as probably the most reliable. ( 1 hartree/ $\mathrm{bohr}^{2}=1.557 \times 10^{6} \mathrm{erg} / \mathrm{cm}^{2}$.)

\begin{tabular}{lcccccccccc}
\hline \hline & LDA & old WVI & FHNC & DMC & RPA & TDLDA & GGA & Meta-GGA & RPA+ & new WVI \\
\hline 2.00 & 3354 & 3527 & & & 3467 & 3533 & 3261 & 3402 & 3413 & 3436 \\
2.07 & 2961 & & 3347 & 3152 & 3064 & 3125 & 2881 & 3002 & 3015 \\
2.30 & 2019 & & 2376 & & 2098 & & 1962 & 2048 & 2060 & 2075 \\
2.66 & 1188 & & 1452 & 1394 & 1240 & & 1152 & 1205 & 1214 & 1218 \\
3.00 & 764 & 813 & & & 801 & 840 & 741 & 779 & 781 & 784 \\
3.28 & 549 & & 719 & 719 & 579 & & 531 & 560 & 563 & 565 \\
4.00 & 261 & 281 & 377 & 390 & 278 & 295 & 252 & 266 & 268 & 270 \\
5.00 & 111 & 121 & 178 & & 119 & 130 & 107 & 113 & 113 & 115 \\
6.00 & 53 & 58 & & & 58 & 65 & 52 & 55 & 54 \\
mrd $(\%)$ & -2.1 & +5.4 & +29.5 & +23.2 & +3.2 & +10.0 & -4.9 & -0.2 & 0.0 & 55 \\
Ref. & 3 & 13 & 4 & 5 & 7 & 7 & 3 & 3 & 9 \\
\hline \hline
\end{tabular}

exchange-correlation hole around an electron. They also found that the exchange $\sigma_{x}$ and correlation $\sigma_{c}$ terms of $\sigma_{x c}$ have canceling small- $k$ (long-range) contributions, so that LDA works better for the sum $\sigma_{x c}$ than for either term separately. Interpolating to the exact $k \rightarrow 0$ asymptote of $\gamma_{x c}(k)$, they found a positive correction to LDA which was $5 \%$ or $10 \%$ of the positive $\sigma_{x c}^{L D A}$ [old wave-vector interpolation (WVI), Table I]. The work of Langreth and Perdew explained why simple density functionals work, and led to the generalized gradient approximations (GGA's), ${ }^{2}$

$$
E_{x c}^{G G A}[n]=\int d^{3} r n \varepsilon_{x c}(n, \nabla n) .
$$

However, the jellium surface energy has remained a puzzle.

The exchange-correlation energy may be written ${ }^{13}$ as

$$
E_{x c}=\frac{1}{2} \int d^{3} r \int d^{3} r^{\prime} \frac{n(\mathbf{r}) n_{x c}\left(\mathbf{r}, \mathbf{r}^{\prime}\right)}{\left|\mathbf{r}-\mathbf{r}^{\prime}\right|},
$$

in atomic units $\hbar=m=e^{2}=1$. Here $n(\mathbf{r})$ is the electron density and $n_{x c}\left(\mathbf{r}, \mathbf{r}^{\prime}\right)$ is the density at $\mathbf{r}^{\prime}$ of the exchangecorrelation hole around an electron at $\mathbf{r}$. The local density $n$ (r) determines the on-top $\left(\left|\mathbf{r}^{\prime}-\mathbf{r}\right| \rightarrow 0\right)$ hole density, not exactly but to a good approximation, ${ }^{14}$ and the low-order derivatives of $n(\mathbf{r})$ determine the short-range (small $\mid \mathbf{r}^{\prime}$ $-\mathbf{r}$ ) behavior of the hole. ${ }^{15}$ By Fourier analyzing the Coulomb interaction $1 /\left|\mathbf{r}^{\prime}-\mathbf{r}\right|$, we find the wave-vector analysis of $E_{x c}$, which we separate into bulk and surface terms.

The solid we consider is jellium, a rigid uniform positive background of density $\bar{n}=3 / 4 \pi r_{s}^{3}=k_{F}^{3} / 3 \pi^{2}$, filling the halfspace $x<0$ and neutralized by electrons. Jellium is a simplified model for simple metals. The surface exchangecorrelation energy is

$$
\sigma_{x c}=\int_{-\infty}^{\infty} d x n(x)\left\{\varepsilon_{x c}([n] ; x)-\varepsilon_{x c}^{u n i f}(\bar{n})\right\}
$$

and its wave-vector analysis is

$$
\gamma_{x c}(k)=\int_{0}^{\infty} d u 8 k_{F} u^{2} b_{x c}(u) \sin (k u) /(k u),
$$

where

$$
b_{x c}(u)=\int_{-\infty}^{\infty} d x n(x)\left\{n_{x c}([n] ; x, u)-n_{x c}^{u n i f}(\bar{n} ; u)\right\}
$$

Here $\varepsilon_{x c}^{u n i f}$ and $n_{x c}^{\text {unif }}$ are the exchange-correlation energy per particle and hole density of the uniform gas, and $n_{x c}([n] ; x, u)$ is the spherical average at separation $u$ of the hole density around an electron at $x$.

Langreth and Perdew ${ }^{13}$ evaluated $\gamma_{x c}^{L D A}(k)$ for jellium, and found $\lim _{k \rightarrow 0} \gamma_{x c}^{L D A}(k) \propto k^{2}$, different from the exact limit

$$
\lim _{k \rightarrow 0} \gamma_{x c}(k)=\frac{k_{F}}{4 \pi}\left(\omega_{s}-\frac{1}{2} \omega_{p}\right) k,
$$

where $\omega_{p}=(4 \pi \bar{n})^{1 / 2}$ and $\omega_{s}=\omega_{p} / \sqrt{2}$ are the bulk and surface-plasmon frequencies, respectively. They then interpolated between Eq. (8) at small $k$ and $\gamma_{x c}^{L D A}(k)$ at large $k$. We shall here interpolate between Eq. (8) at small $k$ and $\gamma_{x c}^{G G A}(k)$ at large $k$. In all our calculations, we have used self-consistent LDA densities $n(x)$. Our LDA is the local part of our GGA. ${ }^{2}$ For the GGA exchange hole, we have used the smoothed model of Ref. 16. For the GGA correlation hole, we have used the real-space cutoff construction of Ref. 10, which is smoothed by the integration over $x$.

Figure 1 shows the wave-vector analysis $\gamma_{x}(k)$ of the surface exchange energy $\sigma_{x}$ (no correlation) for $r_{s}=4$ jellium, in both LDA and GGA. The peak at intermediate $k\left(\approx k_{F}\right)$ is lower in GGA, which displays a negative region at small $k$. These GGA features are similar to those of the exact $\gamma_{x}(k)$ (Ref. 17) for a model density profile, confirming our expectation that GGA should improve upon LDA for intermediate $k\left(\approx k_{F}\right)$. As $k \rightarrow 0, \gamma_{x}^{G G A}(k)$ fails, tending to zero and not to the correct negative constant. ${ }^{18}$ (When comparing Fig. 1 to previous wave-vector analyses of $\gamma_{x}^{L D A}$, one should recall that our hole models average out the long-range oscillatory parts, and so are smooth at $k=2 k_{F}$.)

Figure 2 shows the LDA and GGA wave-vector analyses when correlation is included. As $k \rightarrow 0, \gamma_{x c}^{L D A}(k)$ $\propto k^{2} \cdot \gamma_{x c}^{G G A}(k)$ looks linear at small $k$, but that is an illusion 
$\gamma_{x}(k)\left(\mathrm{erg} / \mathrm{cm}^{2}\right)$

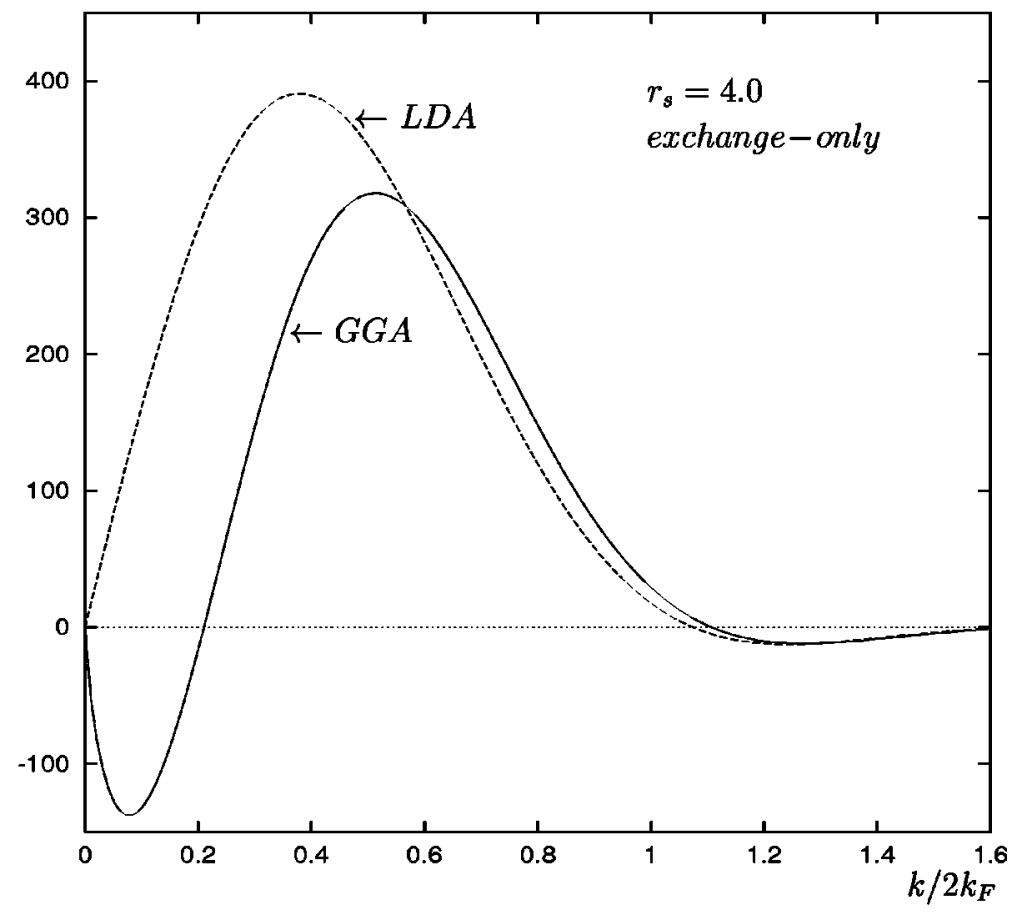

FIG. 1. Wave-vector analysis of the surface exchange energy (no correlation) for the jellium surface with bulk density $r_{s}=4$ bohr. LDA and GGA curves are shown. The area under the curve $\gamma_{x}(k)$ vs $k / 2 k_{F}$ is the surface exchange energy $\sigma_{x}$, in erg $/ \mathrm{cm}^{2}$. The exact $\gamma_{x}(k)$ tends to $-235 \mathrm{erg} / \mathrm{cm}^{2}$ (Ref. 18) as $k \rightarrow 0$. $\left[\sigma_{x}^{L D A}=222, \sigma_{x}^{G G A}=128, \sigma_{x}^{\text {exact }}=157\left(\mathrm{erg} / \mathrm{cm}^{2}\right)\right.$ (Ref. 7).] of scale. The GGA $n_{x c}$ is short ranged for all electron positions, and its $\gamma_{x c}(k)$ is proportional to $k^{2}$ when $k$ is sufficiently close to zero. In comparison with $\gamma_{x c}^{G G A}(k), \gamma_{x c}^{L D A}(k)$ is too high at intermediate $k$, leading to some error in the original interpolation of Langreth and Perdew, ${ }^{13}$ a possibility pointed out by Rasolt et al. ${ }^{17}$

Interpolations from the exact $k \rightarrow 0$ asymptote tend naturally to the peak of $\gamma_{x c}^{G G A}(k)$. We have used the interpolation formula $\gamma_{x c}(k)=a \sin (b x)+c x^{d}$ (where $x=k / 2 k_{F}$ ) for $k$ less than that of the peak. The parameters $a, c$, and $d$ are deter- mined by matching $\gamma_{x c}(k)$ to the exact initial slope at $k$ $=0$, and to the value and vanishing slope of $\gamma_{x c}^{G G A}(k)$ at its peak. This interpolation is also shown in Fig. 2.

The parameter $b$ is chosen to keep the interpolation under control. Figure 2 shows $\gamma_{x c}^{G G A}(k)$ both beyond and within the RPA, the latter constructed from the RPA GGA hole of Ref. 9. These curves differ at large $k$, where the RPA is wrong, and agree at small $k$, where the RPA is right. Overall, they are not so different, consistent with the conclusion ${ }^{8,9}$ that the RPA surface energies require only a small short-range cor-

\section{$\gamma_{x c}(k)\left(\mathrm{erg} / \mathrm{cm}^{2}\right)$}

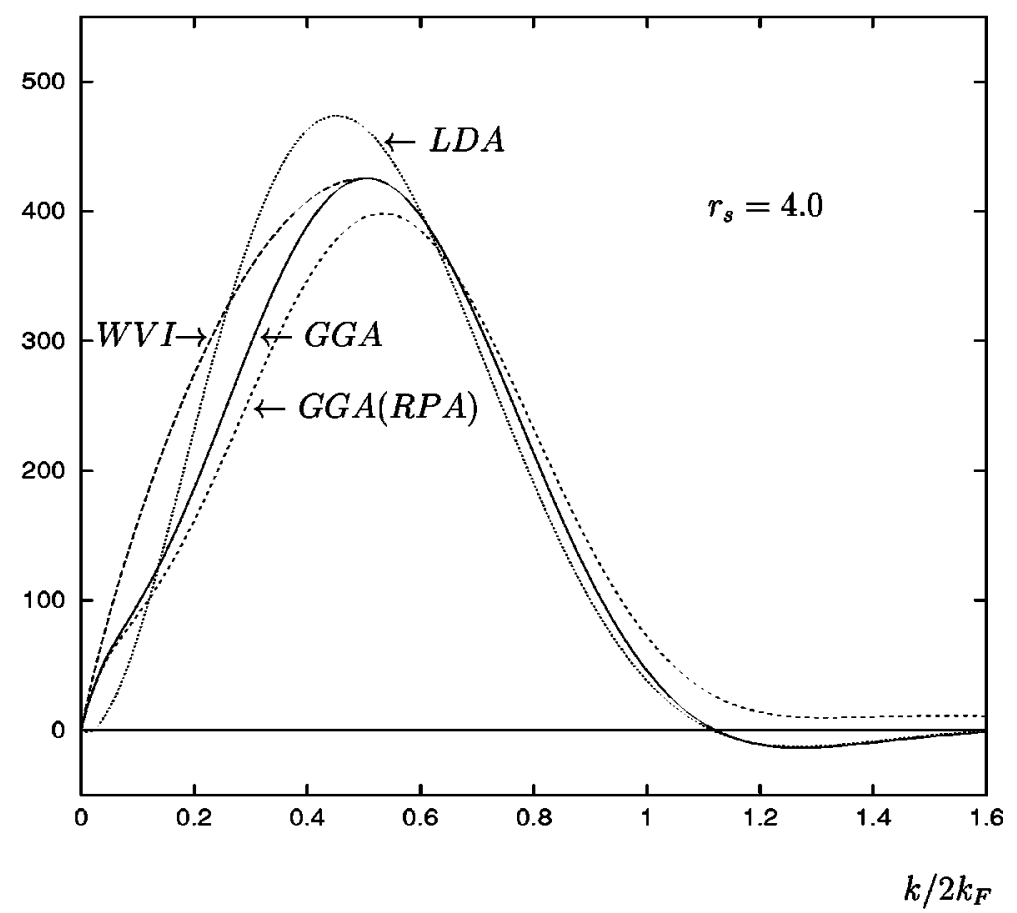

FIG. 2. The wave-vector interpolation (WVI) between the exact $k \rightarrow 0$ asymptote of Eq. (8) and the GGA for intermediate or large wave vectors. Also shown is the GGA within the random-phase approximation (RPA). The parameters for the beyond-RPA interpolation $\left(r_{s}=4\right)$ are $a$ $=1730 \mathrm{erg} / \mathrm{cm}^{2}, b=1.48, c=-1792 \mathrm{erg} / \mathrm{cm}^{2}$, $d=1.28$. 
TABLE II. Total energy per electron ( $E / N$ in eV/electron) for neutral jellium spheres with $N=20$ electrons and bulk density parameter $r_{s}$. All functionals have been evaluated on self-consistent LDA densities. VMC and DMC energies are from Ref. 19. Also shown is the prediction of the liquid drop model (LDM) of Eq. (9), with LDA input; the volume, surface, and curvature terms are shown individually.

\begin{tabular}{ccccccc}
\hline \hline$r_{s}$ & LDA & GGA & Meta-GGA & VMC & DMC & LDM \\
\hline 3.25 & -1.763 & -1.785 & -1.769 & -1.754 & -1.760 & $-1.73=-1.96+0.19+0.04$ \\
4.00 & -1.885 & -1.902 & -1.890 & -1.860 & -1.878 & $-1.86=-2.11+0.21+0.04$ \\
5.62 & -1.794 & -1.803 & -1.795 & -1.738 & -1.782 & $-1.79=-1.99+0.18+0.02$ \\
\hline \hline
\end{tabular}

rection. To fix the parameter $b$, we perform another interpolation from Eq. (8) to the RPA $\gamma_{x c}^{G G A}(k)$, and require that the corresponding $\sigma_{x c}$ of Eq. (2) agree with the RPA calculation of Pitarke and Eguiluz ${ }^{7}$ (the one using LDA orbitals). The result is $b \approx 3.19-0.4286 r_{s}$.

The results of our beyond-RPA interpolation are shown in Table I. Although the interpolated (new WVI) $\sigma_{x c}$ is close to $\sigma_{x c}^{L D A}$, its wave-vector decomposition $\gamma_{x c}(k)$ (Fig. 2) is less like that of LDA (Fig. 2). The LDA $\gamma_{x c}(k)$ has canceling errors at small and intermediate $k$.

The strong long-range contribution $\left[\bar{n}_{x c}(k) \propto k\right.$ according to Eq. (8), or $\bar{n}_{x c}(u) \propto u^{-4}$ as $\left.u \rightarrow \infty\right]$ to the exact surface exchange-correlation energy is unusual. The exact wavevector analysis of $E_{x c}$ for a finite system behaves like $k^{2}$ as $k \rightarrow 0$. This fact explains how (in spin-density functional theory) the GGA can be more accurate than LDA for the atomization energy of a molecule, ${ }^{2}$ and yet less accurate for the metal surface energy. Breaking up a molecule creates extra microsurface around each atom. If there were no anomalous small- $k$ contribution to the exact $\sigma_{x c}$, then LDA would overestimate the metal surface energy and the GGA would correct this overestimation, the typical situation for the atomization energy of a molecule.

The discrepancy between density-functional and diffusion Monte Carlo values for the surface exchange-correlation energy is surprising. Fixed-node DMC results are often regarded as "exact," although those for extended systems require an extrapolation from finite simulation cells. We close this paper with a study of finite jellium spheres, for which no such extrapolation is needed.

${ }^{1}$ R.O. Jones and O. Gunnarsson, Rev. Mod. Phys. 61, 689 (1989).

${ }^{2}$ J.P. Perdew, K. Burke, and M. Ernzerhof, Phys. Rev. Lett. 77, 3865 (1996); 78, 1396(E) (1997), and references therein.

${ }^{3}$ J.P. Perdew, S. Kurth, A. Zupan, and P. Blaha, Phys. Rev. Lett. 82, 2544 (1999); 82, 5179(E) (1999).

${ }^{4}$ E. Krotscheck and W. Kohn, Phys. Rev. Lett. 57, 862 (1986).

${ }^{5}$ P.H. Acioli and D.M. Ceperley, Phys. Rev. B 54, 17199 (1996), and references therein.

${ }^{6}$ N.D. Lang and W. Kohn, Phys. Rev. B 1, 4555 (1970).

${ }^{7}$ J.M. Pitarke and A.G. Eguiluz, Phys. Rev. B 57, 6329 (1998); (private communication).

${ }^{8}$ S. Kurth and J.P. Perdew, Phys. Rev. B 59, 10461 (1999); 60, 11 212(E) (1999).

${ }^{9}$ Z. Yan, J.P. Perdew, and S. Kurth (unpublished).

${ }^{10}$ J.P. Perdew, K. Burke, and Y. Wang, Phys. Rev. B 54, 16533 (1996); 57, 14 999(E) (1998).

${ }^{11}$ H.L. Skriver and N.M. Rosengaard, Phys. Rev. B 46, 7157 (1992).
We consider a neutral jellium sphere with $N=20$ electrons. Table II shows the total energy per electron $E / N$ (including the electrostatic self-energy of the uniform positive background) evaluated in LDA, GGA, and meta-GGA, in comparison with variational (VMC) and diffusion Monte Carlo (DMC) results of Ballone et al. ${ }^{19}$ All three density functionals give similar energies in reasonable agreement with the DMC values, and not much lower as one would expect if the true surface energy were much higher than its density-functional estimates. In particular, the LDA and meta-GGA energies are very close to DMC. The energy of this small cluster is predicted reasonably well by the liquid drop model, ${ }^{20}$ using LDA input ${ }^{21}$ for the surface energy $\sigma$ and curvature energy $\gamma$,

$$
E \approx \varepsilon^{b u l k} N+\sigma 4 \pi R^{2}+\gamma 2 \pi R,
$$

where $R=N^{1 / 3} r_{s}$ is the cluster radius (Table II). While the curvature contribution is small, the surface contribution is not. If DMC surface energies from Table I were used instead of the LDA, the surface term for $r_{s}=4$ would increase from 0.21 to $0.38 \mathrm{eV}$, and the accuracy of Eq. (9) would be lost. In other words, the liquid drop model gives jellium cluster energies consistent with those of DMC, but only if one uses the density-functional estimate for the jellium surface energy.

This work was supported in part by the U.S. National Science Foundation under Grant No. DMR 98-10621, and by the Petroleum Research Fund under Grant No. ACS-PRF No. 33001-AC6. We thank P. Ballone for the refined DMC values of Table II.

${ }^{12}$ L. Vitos, A.V. Ruban, H.L. Skriver, and J. Kollár, Surf. Sci. 411, 186 (1999).

${ }^{13}$ D.C. Langreth and J.P. Perdew, Phys. Rev. B 15, 2884 (1977).

${ }^{14}$ K. Burke, J.P. Perdew, and D.C. Langreth, Phys. Rev. Lett. 73, 1283 (1994).

${ }^{15}$ Y. Wang, J.P. Perdew, J.A. Chevary, L.D. MacDonald, and S.H. Vosko, Phys. Rev. A 41, 78 (1990).

${ }^{16}$ M. Ernzerhof and J.P. Perdew, J. Chem. Phys. 109, 3313 (1998).

${ }^{17}$ M. Rasolt, G. Malmstrom, and D.J.W. Geldart, Phys. Rev. B 20, 3012 (1979).

${ }^{18}$ D.C. Langreth and J.P. Perdew, Phys. Rev. B 26, 2810 (1982).

${ }^{19}$ P. Ballone, C.J. Umrigar, and P. Delaly, Phys. Rev. B 45, 6293 (1992). In Table II, we show refined DMC values from P. Ballone (private communication).

${ }^{20}$ J.P. Perdew, Y. Wang, and E. Engel, Phys. Rev. Lett. 66, 508 (1991).

${ }^{21}$ C. Fiolhais and J.P. Perdew, Phys. Rev. B 45, 6207 (1992). 